Pure \& Appl. Chem., Vol. 57, No. 2, pp. 273-282, 1985.

Printed in Great Britain.

(C) 1985 IUPAC

\title{
Theories of solubilities of non-electrolytes
}

\author{
Saul Goldman \\ The Guelph-Waterloo Centre for Graduate Work in Chemistry, \\ Guelph Campus, Guelph, Ontario, Canada N1G 2W1
}

\begin{abstract}
A brief history of solubility theory is outlined, and the distinguishing features of modern solubility theory are discussed. It is shown how the perturbation theory-based terms in the expression for the Henry's law constant provide microscopic information on covitation and solvation. Results are given both for atomic and molecular systems. In the case of atomic systems, where no adjustable parameters are used, excellent agreement with experiment is found. In these systems we also probe in a fundamental way for the role of quantum effects and nonadditive three-body forces on solubility. For molecular systems, in which the required pair potentials are generally unknown, a somewhat different approach is perhaps most useful. In particular we show how a molecular liquid state perturbation theory can be used to probe for the role of dipoles and quadrupoles on the thermodynamics on non-electrolyte solubilities in water. Finally, some of the difficulties that lie ahead in the field of solubility theory are mentioned.
\end{abstract}

\section{ATOMIC SYSTEMS}

\section{Introduction}

The distinguishing feature of modern solubility theory is that it is based on calculations that explicitly include a function called the radial distribution function. This function, given the symbol $\mathrm{g}(\mathrm{r}, \rho, \mathrm{T})(\mathrm{r}=$ interparticle distance, $\rho=$ number density, $\mathrm{T}=$ absolute temperature), is the factor that multiplies the bulk or microscopic density of a liquid $\rho$ to give the time-averaged microscopic or local density, $\rho g(r, \rho, T)$, around some fixed molecule. The gross characteristics of this function are more or less obvious; i.e. $g(r) \rightarrow$ 0 as $r \rightarrow 0$ since the intermolecular forces through which atoms or molecules interact become increasingly repulsive as the electron clouds around the species begin to overlap. Also, since we are not dealing with solids, which are characterized by long-range order, it should be clear that $\mathrm{g}(\mathrm{r}) \rightarrow 1$ as $\mathrm{r} \rightarrow \infty$. That is, the capacity of a selected molecule to influence the time-averaged microscopic density diminishes as the distance away from the selected molecule becomes large. At intermediate distances this function goes through a number of extrema, the details of which depend on the intermolecular forces in question. As will be shown later, the reason the radial distribution function is so important is that it is always intimately involved in any statistical mechanical theory whose purpose is to calculate the thermodynamic properties of solutions from intermolecular forces.

Almost half a century ago, Sisskind and Kasarnowski (1) published a paper that contained what turned out to be a very clever and insightful idea. They suggested that the energetics involved in the transfer of a molecule from the gas phase to a liquid solution be mathematically decomposed into two terms. One of these terms, they suggested, originates from the work that has to be done against the intermolecular forces in the solvent to create a cavity that will accommodate the solute about to be transferred. This term represents an energy requirement. A second term, representing an energy gain, arises due to the interaction of the solute molecule in the cavity with the surrounding solvent molecules. A few years after its publication, Uhlig (2) and Eley (3) pursued this idea in their work on solubility. Uhlig correlated the surface tensions of a variety of pure liquids with the solubilities of gases in these liquids. Uhlig used the macroscopic surface tension of a liquid to calculate the work required to create a cavity. Eley's approach differed from Uhlig's principally in the way in which he calculated the cavity term. Eley used macroscopic solvent compressibilities for this calculation. For a number of reasons, neither Uhlig nor Eley could come up with quantitative predictions of solubility. These include the inappropriateness of macroscopic surface tensions or compressibilities in the calculation of the work required to make microscopic cavities and the unavailability (at the time) of accurate pair potentials and radial distribution functions, both of which are needed to calculate the second of the two terms - the solute-solvent interaction term.

The next major advance was made about 25 years later by Robert Pierotti (4), who took the same general approach as Sisskind, Kasarnowski, Uhlig, and Eley, but used a newly developed statistical-mechanical theory called the scaled particle theory (Ref.5) (SPT) to calculate the cavity term. Pierotti equated the cavity term in a real solution to the reversible work 
required to create a cavity in the dense assembly of hard spheres. The scaled particle theory provides explicit expressions for the thermodynamic functions that characterize dense hard-sphere or hard convex-like molecule assemblies. But there still remained two fundamental problems with Pierotti's approach. First, a real solution is not comprised of hard spheres, so that Pierotti's estimate of the cavity term is only an estimate. Second, and perhaps more serious, theoretical expressions for the distribution functions were unavailable when Pierotti did his work. Thus Pierotti was forced to approximate $g(r, \rho, T)$ by unity, and therefore he was unable to calculate the contribution made by the solute-solvent interaction term in a fundamental way. Further on in this article we show how the cavity and interaction terms can be accounted for in a rigorous way.

By the early 1970s, all the ingredients needed for a truly fundamental solubility theory (applicable to isotropic systems) had become available. These ingredients included: (1) accurate intermolecular pair potentials obtained from the gas phase by using molecular beams, gas viscosities, and virial coefficients and from solid-state studies; (2) detailed knowledge of the radial distribution function in some prototype of a dense isotropic fluid (The development of Monte Carlo simulations, molecular dynamics calculations, and the solution of integral equations such as the Percus-Yevick equation for dense fluids provided this information. The prototype fluid was the dense hard-sphere fluid and the above methods provided the needed distribution functions, both for pure hard-sphere assemblies and for hard-sphere mixtures); (3) a procedure for connecting the properties of a hardsphere system to those of the system of interest. This connection was provided in a rigorous way by a procedure called either thermodynamic perturbation theory or liquid-state perturbation theory, the salient features of which are outlined below.

\section{Thermodynamic Perturbation Theory}

In the late 1960s Verlet, using computer simulations of hard-sphere assemblies, showed that the structure of a hard-sphere fluid was quite similar to that of a real isotropic fluid in which attractive forces exist (Ref.6). This result and others like it led to the view that the structure, i.e. $g(r, \rho, t)$, of dense isotropic liquids is determined primarily by the repulsive part of the pair potential. Consequently, the influence of the attractive part of the potential on the thermodynamic properties of a fluid can be obtained by a perturbation expansion around a hard-sphere reference state. The required formalism had been previously provided by Zwanzig ( 7 ). The first-order result provided by this theory is given by Eq. 1,

$$
A=A_{0}+2 \pi N \rho \int_{0}^{\infty} r^{2} u_{p}(r) g_{o}(r) d r+\ldots
$$

where $A$ and $A_{0}$ are the Helmholtz free energy in the real and reference system respectively, $g_{o}(r)$ is the radial distribution function in the reference state, and $u_{p}(r)$, the perturbing pair potential, is given by eq 2 . In eq $2 u(r)$ and $u_{0}(r)$ are the actual and reference

$$
u_{p}(r)=u(r)-u_{o}(r)
$$

state intermolecular pair potentials, respectively.

The infinite series represented by Eq. 1 will converge rapidly (i.e., the first two terms shown in the series will closely approximate $A)$ if $B u_{p}(r)$ is small, $i . e .$, if the temperature is high relative to the perturbing potential. It can also be made to converge rapidly at low temperatures and high densities if the division of $u(r)$ into $u_{p}(r)$ and $u_{o}(r)$ is such that $g_{o}(r)$ closely approximates $g(r)$. Since, as already mentioned, the structure of a dense isotropic liquid is determined primarily by the repulsive part of the pair potential, $g_{0}(r)$ will closely approximate $g(r)$ at high densities if $u_{0}(r)$ is based on the repulsive part of the pair potential. We will return to this point further on. The higher order terms in the series are complicated and difficult to evaluate accurately because they involve integrals over higher order reference-state distribution functions (e.g., triplet, quadruplet...distribution functions) about which little is known. This is one of the problems encountered in the direct application of Zwanzig's theory to liquids. Another is the problem of how to separate $u(r)$ into $u_{o}(r)$ and $u_{p}(r)$.

For these reasons three different perturbation theories, similar in spirit but different in details from Zwanzig's, have been developed (Refs.8-13). They all provide an objective basis for the separation discussed above, and also they were formulated to ensure rapid convergence of the series shown in Eq. 1 .

Before describing these theories it is worthwhile to mention one additional problem that occurs when perturbation theory is applied to mixtures as opposed to pure fluids. If we consider a binary solution in which the solute has a significantly deeper well depth than the solvent, then the solute may tend to form clusters or aggregates because of the attractive part of its pair potential, so that for this situation the first two terms in Eq. 1 will provide an inadequate approximation for $\mathrm{A}$, and higher order terms have to be included (Refs.14,15). Fortunately this problem is not strongly relevant to the present 
application wherein the solute is always at infinite dilution and so cannot form clusters.

The first-order expressions for binary mixtures that obey classical statistics with pairwise additive intermolecular potentials are given by Eqs. 3-5 for the Leonard-Henderson-

$$
\begin{aligned}
& B f=\beta f^{O}-4 \pi \rho x_{1} d_{12}{ }^{2} g_{12}{ }^{o}\left(d_{12}\right)\left[d_{12}-\delta_{12}\right]+2 \pi \rho \beta \sum_{i j=1}^{2} x_{i} x_{j} \int_{\sigma_{i j}}^{\infty} u_{i j} p(r) g_{i j}{ }^{o}(r) r^{2} d r \\
& \beta f=\beta f^{O}+2 \pi \rho \beta \sum_{i j=1}^{2} x_{i} x_{j} \int_{d i j}^{\infty} u_{i j} p(r) g^{o} i j(r) r^{2} d r \\
& B f=\beta f^{O}+2 \pi \rho \beta \sum_{i j=1}^{2} x_{i} x_{j} \int_{0}^{\infty} u_{i j} p(r) \exp \left[-\beta u_{i j}^{o}(r)\right] y_{i j}{ }^{H S}(r) r^{2} d r
\end{aligned}
$$

Barker $(8,9)$, variational or Mansoori-Canfield $(10,11)$, and Weeks-Chandler-Andersen $(12,13)$ theories, respectively. In each of these equations the division of $u(r)$ into $u_{p}(r)$ and $u_{0}(r)$ is different (see below), so that the terms $\beta f^{0}, u_{i j}(r)$, and $g_{i j}^{o}(r)$ are all different. Hereafter we will use the abbreviations LHB, MC, and WCA for these theories. In these equations

$$
B f \equiv \frac{A\left(N_{1}, N_{2}, V, T\right)-A^{i g}\left(N_{1}, N_{2}, V, T\right)}{\left(N_{1}+N_{2}\right) k T}
$$

is the reduced Helmholtz free energy per particle relative to this quantity in an ideal gas mixture at the same $N_{1}, N_{2}, V$, and $T$ as the solution of interest. $B f$ is this quantity for the reference state mixture. Also $x$ is mole fraction, $\rho$ is total number density $=\left(N_{1}+\right.$ $\mathrm{N}_{2}$ )/V, the superscripts $o, p$, and HS mean reference state, perturbation, and hard sphere, respectively, $d_{i j}$ is the hard-sphere reference-state diameter between particles $i$ and $j$, and $y_{i j}(r)$ is the hard-sphere radial distribution function, smoothly extrapolated to $\mathrm{r}=0$. The term $\delta_{12}$ in $\mathrm{Eq} .3$ is similar in magnitude to $\mathrm{d}_{12}$.

The LHB and MC theories are both based on a hard-sphere reference state, but the way in which the hard-sphere diameters are calculated differ (Refs.8,11) The WCA theory is not based on hard-sphere reference state. Rather it is obtained from the following division of the potential $u(r)$

$$
\begin{aligned}
u_{o}(r) & =u^{L J}(r)+\varepsilon, & & r<r_{m} \\
& =0, & r & >r_{m} \\
u_{p}(r) & =-\varepsilon, & r & <r_{m} \\
& =u^{L J}(r), & r & >r_{m}
\end{aligned}
$$

where $u^{L J}(r)$ is the Lennard-Jones 6-12 pair potential (Ref.16) and $r_{m}$ the position of minimum potential. In this particular division $u_{0}(r)$ is based on all the repulsive forces in the Lennard-Jones system. Consequently $g_{o}(r)$ will very closely resemble $g(r)(\operatorname{Ref} .17)$, so that the first two terms in eq 1 will provide a particularly good approximation for A.

\section{Thermodynamics}

We now connect the measurable quantities that arise in solubility investigations with the statistical-mechanical quantity $B f$ which is calculable from any one of Eqs. 3 to 5 .

The most commonly used measure of solubility of a volatile solute is the Henry's law constant. For a vapor phase over a solution that is reasonably dilute (i.e., $\mathrm{P} \lesssim 10 \mathrm{~atm}$ ) the solute in the vapor phase may be considered to behave as an ideal gas. Under these circumstances the Henry's law constant $(\mathrm{K})$ of the solute is given by $\mathrm{Eq}$. 6 , where $\mathrm{P}_{2}$ is the partial pressure of the solute over the solution and $x_{2}$ is the mole fraction of the solute

$$
\mathrm{K}=\lim _{\mathrm{x}_{2} \rightarrow 0}\left(\mathrm{P}_{2} / \mathrm{x}_{2}\right)
$$

in the solution. From this definition and the usual thermodynamic relations we obtain (Ref.18) Eqs. 7-9. In these equations $R$ is the gas constant, subscripts 2 and 1 mean solute and solvent, respectively, $\overline{\mathrm{V}}_{1}$ is the molar volume of the pure solvent, $n$ denotes number of moles, and the superscript ${ }^{-1 e n o t e s}$ the limit $\mathrm{x}_{2} \rightarrow 0 ; \Delta \mathrm{H}{ }^{\circ}$ and $\overline{\mathrm{V}}_{2}$ are respectively the molar heat of solution and the partial molar volume of the solute at infinite dilution. 


$$
\begin{gathered}
\ln \mathrm{K}=\ln \left(\frac{\mathrm{RT}}{\overline{\mathrm{V}}_{1}}\right)+(\beta \mathrm{f})^{\bullet}+\mathrm{n}_{1}\left(\frac{\partial \beta \mathrm{f}}{\partial \mathrm{n}_{2}}\right)_{\mathrm{n}_{1}, \mathrm{~T}, \mathrm{~V}} \\
{\Delta \mathrm{H}_{\mathrm{S}}}^{\bullet}=-\mathrm{RT}^{2}\left(\frac{\partial \ln \mathrm{K}}{\partial \mathrm{T}}\right)_{\mathrm{P}} \\
\overline{\mathrm{v}}_{2} \cdot=\operatorname{RT}\left(\frac{\partial \mathrm{ln} \mathrm{K}}{\partial \mathrm{P}}\right)_{\mathrm{T}}
\end{gathered}
$$

Microscopic Basis of Cavity Term and Solute-Solvent Interaction Term

We have in Eq. 7 a connection between solubility and terms that directly involve the energetics of the solution process at a microscopic level. For example, if Eq. 4 from the variational theory is used for $\beta f$ in Eq. 7, we obtain (Ref.19,20) Eq. 10,

$$
\ln \mathrm{K}=\ln \left(\frac{\mathrm{RT}}{\mathrm{V}_{1}}\right)+\beta \mu_{2}{ }^{\cdot \mathrm{HS}}+\mathrm{I}_{11}{ }^{\bullet}+\mathrm{I}_{12} \cdot
$$

where

$$
\begin{aligned}
& \beta \mu_{2} \cdot \mathrm{HS}=\beta \mathrm{f}^{\bullet \mathrm{HS}}+\mathrm{N}_{1}\left(\frac{\partial \mathrm{ffHS}}{\partial \mathrm{N}_{2}}\right)_{N_{1}, \mathrm{~T}, \mathrm{~V}}^{*} \\
& I_{11}=2 \pi \rho N_{1} \beta \frac{\partial}{\partial N_{2}}\left(\int_{d_{11}}^{\infty} r^{2} u_{1 l}(r) g_{11} H^{H}(r) d r\right)_{N_{1}, T, V}^{0} \\
& \dot{I}_{12}=4 \pi \rho_{1} \beta \quad \int_{d_{12}}^{\infty} r^{2} u_{12}(r) g_{12}{ }^{H S}(r) d r
\end{aligned}
$$

The $\ln \left(\mathrm{RT} / \overline{\mathrm{V}}_{1}\right)$ term arises solely because of a different choice for the solute standard state in the liquid and gas phases, so. that it is not involved in either the cavity or the interaction terms. The term $\beta_{\mu} \cdot \mathrm{AS}^{2}$, on the other hand, comes from the reversible work required to create a cavity of diameter $d_{22}$ in a hard-sphere system of particles with diameter $d_{11}$ at the same density, temperature, and composition (infinite dilution with respect to the solute) as the solution of interest. Clearly, however, there has to be an additional term that corrects the hard-sphere cavity term to a cavity term for the softsphere system of interest. It was first pointed out by Neff and McQuarrie (21) that the term represented by $I_{11}$ provides this correction to the first order. As may be seen by examining the expression for $\mathrm{I}_{11}{ }^{\circ}$, this term arises because of a change in the total solvent-solvent interaction potential energy beyond that accounted for by hard-sphere potentials. This change is brought about by the creation of a cavity in a real solvent. The presence of the cavity causes the solvent structure to be altered from what it is in the absence of a cavity, and this results in a change in the total solvent-solvent interaction potential energy. Finally, the solute-solvent interaction energy is accounted for by the term $I_{12}$. The fact that this term has the form of the integral shown has long been known. What is new is our abilit $y_{\mathrm{H}} 5^{\circ}$ obtain accurate values of $\mathrm{I}_{12}$ because of our newly acquired knowledge of the term $\mathrm{g}_{12} \mathrm{HS}(\mathrm{r})$ in the integrand of this term.

\section{Results}

Relative and Absolute Accuracy of the Theories

Each of Eq. 3 to 5 is based on a different perturbation theory. Consequently, by using each of these equations in Eq. 7 to 9 and applying the result to a particular system, we can intercompare the three theories with respect to how well they predict experimental results, and we can learn how sensitive a particular solubility function (e.g., $\mathrm{K}, \Delta \mathrm{H}_{\mathrm{s}}{ }^{\circ}$, or $\overline{\mathrm{V}}_{2}{ }^{\circ}$ ) is to the theory being used to obtain it. This was done for a variety of systems, (Ref.22) and a sample of the results obtained are displayed in Table 1 for the system Ne in Ar. In these calculations the same intermolecular pair potentials, taken from gas-phase virial coefficients, were used. We see from Table 2 that the LHB and WCA theories provide similar and good predictions for $\mathrm{ln} \mathrm{K}$, but that the predictions from the MC theory are worse. The relatively poor performance of the MC theory is believed due to the fact that the upper bound on the free energy that this theory is based on (Refs.10,11) is too high a bound to be useful for highly accurate solubility predictions. The closeness of the predictions of the other two theories (both to experimental results and to each other) is remarkable. The WCA theory is known to be more accurate than first-order LHB theory with respect to the prediction of total thermodynamic properties (e.g.,Bf) (Ref.23). Apparently the function

$$
(\beta f)^{\bullet}+n_{1}\left(\frac{\partial \beta f}{\partial n_{2}}\right)^{\cdot}
$$

upon which solubility depends (see Eq. 7) entails a fair amount of cancellation among the 
higher order terms left out in the LHB theory.

TABLE 1 Comparisons of Calculated and Experimental Values of $\ln \mathrm{K}, \Delta \mathrm{H}_{\mathbf{s}}{ }^{*}$, and $\overline{\mathrm{v}}_{2}$ for $\mathrm{Ne}$ in $\mathrm{Ar}$

\begin{tabular}{|c|c|c|c|c|c|c|c|c|c|c|c|c|}
\hline \multirow[b]{2}{*}{$\mathrm{T}, \mathrm{K}$} & \multicolumn{4}{|c|}{$\ln (\mathrm{K} / \mathrm{atm})$} & \multicolumn{4}{|c|}{$\Delta_{\mathrm{S}}{ }^{\bullet}, \mathrm{cal} / \mathrm{mol}$} & \multicolumn{4}{|c|}{$\overline{\mathrm{V}}_{2}{ }^{\bullet}, \mathrm{cm}^{3} / \mathrm{mol}$} \\
\hline & LHB & MC & WCA & $\operatorname{expt} 1^{a}$ & LHB & MC & WCA & $\operatorname{expt} 1^{a}$ & LHB & MC & WCA & $\operatorname{expt1}{ }^{a, b}$ \\
\hline $\begin{array}{r}87.29 \\
87.42 \\
115.80\end{array}$ & $\begin{array}{l}6.77 \\
6.77 \\
6.48\end{array}$ & $\begin{array}{l}7.40 \\
7.40 \\
6.88\end{array}$ & $\begin{array}{l}6.93 \\
6.92 \\
6.56\end{array}$ & $\begin{array}{l}6.99 \\
6.97 \\
6.48\end{array}$ & $\begin{array}{l}132 \\
132 \\
437\end{array}$ & $\begin{array}{l}288 \\
289 \\
627\end{array}$ & $\begin{array}{l}159 \\
159 \\
461\end{array}$ & $\begin{array}{l}201 \\
232 \\
597\end{array}$ & $\begin{array}{l}23.1 \\
23.2 \\
40.5\end{array}$ & $\begin{array}{l}27.2 \\
37.3 \\
47.3\end{array}$ & $\begin{array}{l}26.1 \\
26.1 \\
43.8\end{array}$ & $\begin{array}{r}25 \\
- \\
45\end{array}$ \\
\hline
\end{tabular}

a These values are from W.B. Streett (Ref.40); the $\Delta_{H^{*}}$ have an estimated uncertainty of 150 cal/mol (Ref.4). These values are from W.B. Streett, (Ref.41); their uncertainty is estimated to be $5 \mathrm{~cm}^{3} / \mathrm{mol}$.

We see from Table 1 that all three theories predict the functions ${\Delta \mathrm{H}_{\mathrm{s}}}^{\cdot}$ and $\overline{\mathrm{V}}_{2}{ }^{\bullet}$ to within experimental error but there is a sizeable uncertainty in the experimental values of these functions. The spread in the predicted values of these quantities that results from the use of the different theories is certainly significant; presumably the WCA predictions are the most accurate.

The Influence of Quantum Effects (Ref.20)

It is easy to show, on the basis of the much smaller mean free path in liquids than in gases, that conditions which ensure the validity of classical statistical mechanics are much more readily found in the gas phase than in solution (Ref.20). This difference in the degree of validity of classical statistics makes it necessary to correct the theoretical solubility expressions based on classical statistics for those systems in which the temperature is low $(\mathrm{T}<150 \mathrm{~K}$ ) or the solute is light (i.e., molecular mass $<20$ ) or both. For systems in which the quantum deviations are not too large (e.g., excluding solutions involving $\mathrm{H}_{2}$ or He near absolute zero) the deviations can be treated as a perturbation on the classical result. The detailed expressions for the first-order quantum correction to the Helmholtz free energy and the chemical potential have been given elsewhere,(Ref.20) and here we focus on some of the results that were found.

It is seen from Figs. 1 and 2 and Table 2 that the effect of including the correction for quantum deviations is to raise the calculated values of $\mathrm{K}, \Delta \mathrm{H}$, and $\mathrm{V}_{2}$ relative to what is obtained on the basis of purely classical statistical mechanics. More specifically, we see from Fig. 1 that the inclusion of the quantum term is crucial for quantitative agreement with experiment when the solute is $\mathrm{H}_{2}$ and the solvent is either liquid $\mathrm{N}_{2}$, $\mathrm{Ar}$, or $\mathrm{CH}_{4}$. We see from Fig. 2 that the quantum correction for Ne in either liquid $\mathrm{N}_{2}$ or Ar is significant at the lower temperatures shown but that it becomes less important as the temperature goes up.

Table 2 demonstrates the result that for $\mathrm{H}_{2}$ in benzene at ordinary temperatures the quantum term contribution to $\mathrm{K}$ and to $\Delta \mathrm{H}$ cannot be neglected since it contributes between 5 and $10 \%$ toward the overall value of these functions. The quantum correction to $\overline{\mathrm{V}}_{2}{ }^{\circ}$ is relatively small. Table 2 also demonstrates the result that quantum deviations in solubility calculations can be neglected in ordinary solvents at ordinary or high temperatures provided the solute has a molecular mass equal to or greater than that of He. This limit was never before established.

TABLE 2 Deviations between the Classically Calculated Values of $\mathrm{K} \overline{\mathrm{V}}_{2}{ }^{\bullet}$, and $\Delta_{\mathrm{H}}{ }^{\bullet}(\mathrm{cal} / \mathrm{mol})$ and the Corresponding Quantum-Corrected Values for $\mathrm{H}_{2}$, He, and $\mathrm{Ne}$ in $\mathrm{C}_{6}^{\mathrm{S}} \mathrm{H}_{6}$

\begin{tabular}{|c|c|c|c|c|c|c|c|c|c|}
\hline \multirow[b]{2}{*}{$t,{ }^{\circ} \mathrm{C}$} & \multicolumn{3}{|c|}{$\mathrm{K}($ quantum $) / \mathrm{K}(\text { classical })^{\mathrm{a}}$} & \multicolumn{3}{|c|}{ 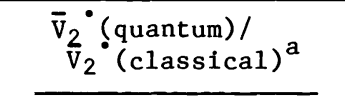 } & \multicolumn{3}{|c|}{$\underset{\Delta \mathrm{H}_{\mathrm{s}}^{\mathrm{s}}}{\Delta \mathrm{H}^{\bullet}} \stackrel{(\text { quantum })-}{(\text { classical })^{\mathrm{a}, \mathrm{b}}}$} \\
\hline & $\mathrm{H}_{2}$ & $\mathrm{He}$ & $\mathrm{Ne}$ & $\mathrm{H}_{2}$ & $\mathrm{He}$ & $\mathrm{Ne}$ & $\mathrm{H}_{2}$ & $\mathrm{He}$ & $\mathrm{Ne}$ \\
\hline 20 & 1.066 & 1.021 & 1.008 & 1.022 & 1.008 & 1.003 & 90 & 29 & 12 \\
\hline 60 & 1.047 & 1.015 & 1.006 & 1.018 & 1.007 & 1.003 & 77 & 25 & 10 \\
\hline 100 & 1.035 & 1.012 & 1.004 & 1.016 & 1.006 & 1.002 & 68 & 22 & 8. \\
\hline 150 & 1.024 & 1.008 & 1.003 & 1.013 & 1.005 & 1.002 & 62 & 20 & \\
\hline 200 & 1.017 & 1.006 & 1.002 & 1.011 & 1.004 & 1.001 & 59 & 19 & \\
\hline
\end{tabular}

a The experimental values of $\mathrm{K}, \overline{\mathrm{V}}_{2}{ }^{\bullet}$, and $\Delta \mathrm{H}_{\mathrm{S}}{ }^{\bullet}$ for $\mathrm{H}_{2}$ in $_{\mathrm{b}} \mathrm{C}_{6} \mathrm{H}_{6}$ at $25^{\circ} \mathrm{C}$ are $3.38 \times 10^{3}$ atm, 35 $\mathrm{cm}^{3} / \mathrm{mol}$, and $1520 \mathrm{cal} / \mathrm{mol}$, respectively (Rets.42,43). The results for the quantum effect on $\Delta \mathrm{H}_{\mathrm{s}}$ are expressed as a difference rather than a ratio, since $\Delta \mathrm{H}_{s}$ for the $\mathrm{H}_{2}$ in $\mathrm{C}_{6} \mathrm{H}_{6}$ system goes through zero in the range $20-200^{\circ} \mathrm{C}$. 
The Role of Non-additivity of the Pair Potentials (Ref.24)

A feature shared by all previous generations of solubility theories was the assumption of perfect additivity of the intermolecular pair potentials. This obviously cannot be perfectly true because the presence of other particles around an interacting pair will, because of their interactions with the pair, alter the pair potential from what it would be in the absence of neighboring particles.

It is generally believed (at least for isotropic particles) that the most important of the higher-body nonadditive terms is that due to three-body dispersion forces, for which the potential energy is given by the Axilrod-Teller formula (Ref.25). As with quantum deviations, three-body nonadditive effects can be formally dealt with by treating them as a perturbation to the two-body (i.e., pairwise additive) result. The detailed form of the first-order correction to $B f$ due to this effect has been given elsewhere (Refs.24,26), and here we will concentrate on the results obtained.

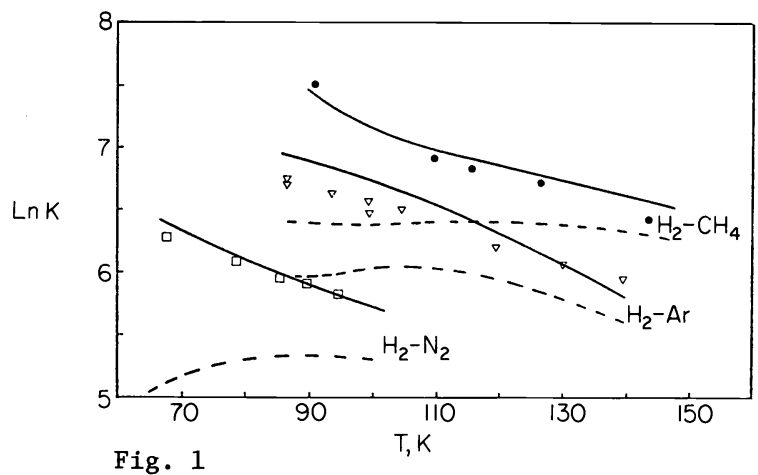

Fig. 1

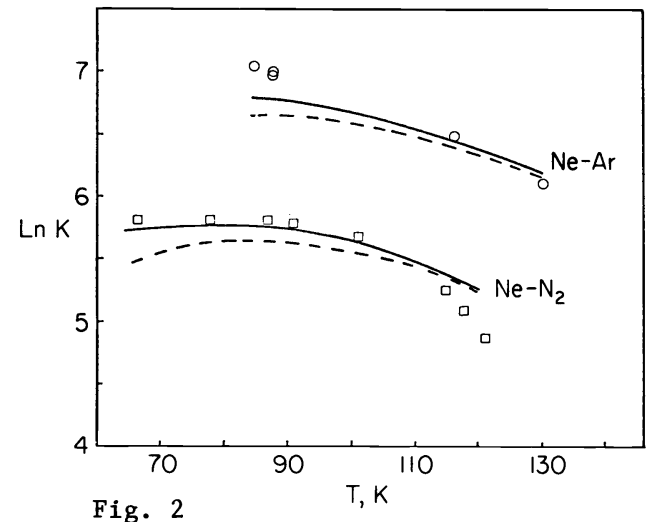

Fig. 2

Fig. 1. Calculated and experimental Henry's law constants for hydrogen. The solid and broken curves give the quantum-corrected and classical results respectively (Ref.44): (•) $\mathrm{H}_{2}$ in $\mathrm{CH}_{4} ;(\nabla) \mathrm{H}_{2}$ in $\mathrm{Ar}$; (口) $\mathrm{H}_{2}$ in $\mathrm{N}_{2}$.

Fig. 2. Calculated and experimental Henry's law constants for neon. Curves are explained in caption for Figure 1. Data sources: $\mathrm{Ne}$ in $\mathrm{Ar},(\operatorname{Ref} .40) ; \mathrm{Ne}$ in $\mathrm{N}_{2}$, (Ref.45); (o) Ne in Ar; (G) $\mathrm{Ne}$ in $\mathrm{N}_{2}$.

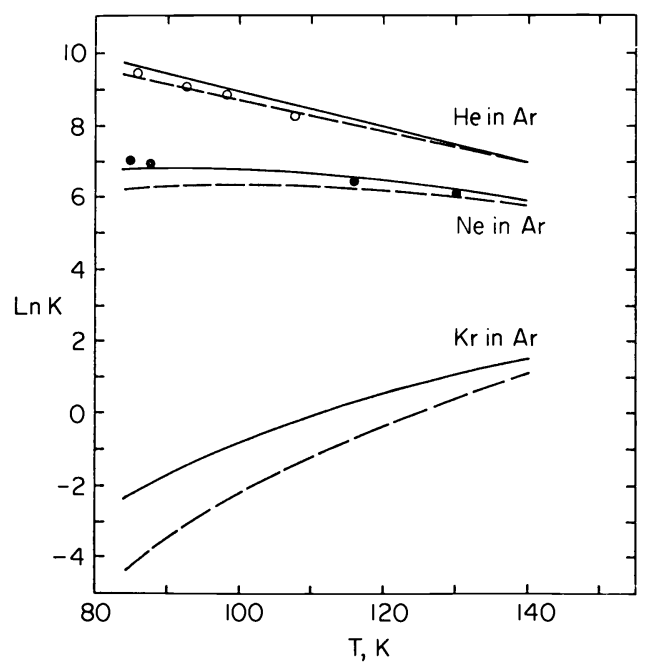

Fig. 3. Calculated and experimental Henry's law constants. The solid curves include the three-body dispersion nonadditive term; the dashed curves do not. Data sources: Ne in $\mathrm{Ar}(\operatorname{Ref.40)}$; He in $\mathrm{Ar}(\operatorname{Ref.46)}$. (O) $\mathrm{Ne}$ in $\mathrm{Ar} ;(\mathrm{O}) \mathrm{He}$ in $\mathrm{Ar}$. 
Some of the results of the calculations for $1 \mathrm{n} \mathrm{K}$ are shown in Fig. 3, from which the following principal features are apparent.

(1) The effect of including the triple-body dispersion term is to raise the calculated values of $\mathrm{K}$ relative to the values obtained for this quantity using only two-body forces.

(2) The relative influence of the three-body term on $\mathrm{K}$ increases in the order (He in $\mathrm{Ar}$ ) < (Ne in $\mathrm{Ar})<(\mathrm{Kr}$ in $\mathrm{Ar})$. The effect is so pronounced in the system $\mathrm{Kr}$ in $\mathrm{Ar}$ that it is unlikely that "effective" pair potentials (which are contaminated by nonadditive effects) could ever properly account for the thermodynamics of this or of any system with a large solute:solvent size ratio. (3) Inclusion of the three-body nonadditive term results in excellent agreement with experimental data in those systems for which data are available. For the system Ne in Ar, where inclusion of this term significantly alters the calculated values of $\mathrm{K}$, the agreement with experiment is improved relative to what is obtained by assuming pairwise additivity. Simịlar conçlusions are drawn by examining the effect of nonadditivity on the functions $\Delta \mathrm{H}_{\mathrm{s}}$ and $\overline{\mathrm{V}}_{2}$ (Ref.24).

\section{MOLECULAR SYSTEMS}

\section{The Approach}

In view of the fact that for practically any molecular system of interest, the full angleand distance-dependent pair potentials are unknown, a somewhat different approach with different goals seems indicated. Thus in the work described below we do not try to numerically duplicate experimental solubilities. Rather, the approach taken is to selectively "turn on" specific types of anisotropy in a solvent, with a view to determining how these anisotropies in a solvent influence solubilities. The kind of question we focus on is: to what extent can for example, a strongly polar, or a strongly quadrupolar solvent, etc., be theoretically expected to manifest hydrophobic-like solvent characteristics.

\section{The Mode1}

The solute is a Lernard-Jones sphere and the solvent is a Lennard-Jones sphere plus one of the following anisotropies: (i) an embedded point dipole (ie a Stockmayer potential, referred to by the abbreviation $L+D$ below) (ii) an embedded point quadrupole ( $L+Q$ below) (iii) an embedded point dipole and a point quadrupole $(L+D+Q)$. We pick the Lennard-Jones parameters for the solute and solvent to be the same, so that all non-ideal effects are due to the electrostatic anisotropy of the solvent-solvent interactions. The size of the Lennard-Jones parameters and the magnitudes of the dipole and quadrupole moments were taken from an electropole model for water (Ref.27). A complete description of this work is given elsewhere (Ref.28).

\section{Molecular Perturbation Theory}

The perturbation theory that was used was the Gray-Gubbins-Stell perturbation theory, the details of which are available in (Ref.29) to (Ref.38).

As with any perturbation theory, the properties of the system of interest are obtained by expanding around a reference system whose properties are presumed known. The anisotropic pair potential is written as a sum of an isotropic and an anisotropic term. The isotropic term, which is the reference state potential, is chosen to be the unweighted angle average of the total potential over the orientations of both molecules. This choice of a reference state was first used by Pople in the earliest version of this theory (Ref.29). The Helmholtz free energy is then expanded in powers of the perturbing potential to give

$$
A=A_{0}+A_{2}+A_{3}+\ldots,
$$

where $A$ is the Helmholtz free energy of the system of interest, $A_{0}$ is the reference state contribution to $\mathrm{A}$,

$$
A_{2}=-\frac{1}{2} B\left\langle U_{a}^{2}\right\rangle_{0}
$$

and

$$
A_{3}=\frac{1}{6} B^{2}\left\langle U_{a}^{3}\right\rangle_{0}
$$

Here $B=1 / \mathrm{kT}, \mathrm{k}$ is the Boltzmann constant, $\mathrm{T}$ the absolute temperature, and $\mathrm{U}_{\mathrm{a}}$ is the anisotropic part of the total configurational energy of the system. In Eq. 11 the firstorder contribution to A vanishes for multipolar anisotropies because of the choice of the reference state. Working expressions for $\mathrm{A}_{2}$ and $\mathrm{A}_{3}$ are obtained by assuming pairwise additivity of the intermolecular potential, expanding the anisotropic part of the pair potential in generalized spherical harmonics, and then using the orthogonality and symmetry properties of the representation coefficients and the Clebsch-Gordan coefficients, to do the angle averaging analytically. For the most general anisotropic potential, $A_{2}$ involves sums of two- and three-body terms, and $A_{3}$ sums of two-, three-, and four-body terms. Carrying Eq. 11 to a higher order would involve even larger many-body terms, for which the required reference state distributions are unknown. 
Equation 11, truncated at the third order, converges slowly for strong multipoles, and is unsuitable for the calculations described below. The development that made this theory applicable here was the Padé approximant to this series proposed by Stell et al., (31) namely,

$$
A=A_{0}+\frac{A_{2}}{1-A_{3} / A_{2}}
$$

This approximant is known to be much more accurate than the third order form of Eq. 11 for strong dipoles, quadrupoles, and mixed dipole-quadrupole interactions. Since $A$ is explicitly calculable by Eq. 12, so too are all the thermodynamic functions through Eqs. 12 and 7 to 9. Thus our approach here is to monitor the response of the solubility-related thermodynamic functions to specific electrostatic anisotropies in the solvent.

Results

The results are presented graphically in Figs. 4-7.

It is clear from Fig. 4, that with respect to the Henry's law constant $K$, at ordinary temperatures, either a purely Lennard-Jones plus dipole ( L+D) or a purely Lennard-Jones plus quadrupole solvent $(L+Q)$ behaves in a very nonhydrophobic way. For purposes of comparison, we note that $1 \mathrm{nK}$ for the rare gas solutes He to $\mathrm{Rn}$ in water at $25^{\circ} \mathrm{C}$ spans the range 11.9 (He) to 8.7 ( $R n$ ) (Ref.39), so that the calculated solubilities for the $L+D$ and $\mathrm{L}+\mathrm{Q}$ solvents are much too high. What is noteworthy, however, is that when we turn on the dipole-dipole, quadrupole-quadrupole, and the two dipole-quadrupole cross terms all simultaneously (the $L+D+Q$ solvent), we get a huge increase in $1 \mathrm{nK}$, especially at ordinary temperatures. This increase is in the right direction with respect to hydrophobic solvation, in that it brings our calculated $1 \mathrm{nK}$ values a lot closer to the range of experimental values. It is important to remember that these differences among the solvents in $1 \mathrm{nK}$, and in the other thermodynamic functions, are entirely a consequence of differences in the permanent electrostatic part of the solvent-solvent pair potentials.

The comparisons in Figs. 5 to 7 are in a sense more revealing. The calculated enthalpies of solution shown in Fig. 5 are, for all three solvents, in the right range for hydrophobic enthalpies of solution; the experimental $\Delta \mathrm{H}^{*}$ values in $\mathrm{kcal} / \mathrm{mol}$ for He to $\mathrm{Rn}$ in water at $25^{\circ} \mathrm{C}$ cover the range $-0.2(\mathrm{He})$ to $-5.1(\mathrm{Rn})$ (Ref.39). Note, however, the difference in the temperature dependence of $\Delta \mathrm{H}_{s}{ }^{\circ}$ for the $\mathrm{L}+\mathrm{D}+\mathrm{Q}$ solvent, relative to the other two solvents. This manifests itself in the very different behavior of the function $\bar{C}_{2}$ for the three solvents (Fig. 6). Now only the $L+D+Q$ solvent gives a $\bar{C}_{2}{ }_{2}$ value in the range characteristic of hydrophobic solvation: $25.1 \mathrm{cal} / \mathrm{mol} \mathrm{K}$ (He) to $69.9 \mathrm{cal} / \mathrm{mol} \mathrm{K}$ (Rn) (Ref.39). Thus, the large positive $\overline{\mathrm{C}}_{2}$, which is characteristic of hydrophobic solvation, cannot be mimicked by a Lennard-Jones solvent containing a large dipole moment only, or a large quadrupole moment only. However, when large dipole-dipole, quadrupole-quadrupole, and the dipole-quadrupole cross terms all act in concert, we do get $\mathrm{Cp}_{2}$ 's in the range found experimentally in water.

As is seen from Fig. 7, a rather different picture emerges for the entropy of solution $\Delta \mathrm{S}_{\mathrm{s}} \cdot$ The experimental values at $25^{\circ} \mathrm{C}$ for the rare gases in water, in entropy units, cover the range -24.2 (He) to $-34.4(R n)$ (Ref.39). Thus, the $L+D+Q$ solvent and the $L+D$ solvent behave similarly with respect to $\Delta \mathrm{S}_{\mathrm{S}}{ }^{\circ}$, in that they both involve large negative entropies of solution that are almost, but not quite, in the experimental range for hydrophobic solutions. The $\Delta \mathrm{S}_{\mathrm{S}}$ obtained for the $\mathrm{L}+\mathrm{Q}$ solvent is much less negative at ordinary temperatures and $\Delta \mathrm{S}_{S}{ }^{\bullet}$ for this solvent rises, rather than falls with temperature, at ordinary temperatures.

\section{DISCUSSION AND SUMMARY}

We have shown how modern liquid state perturbation theory, together with accurately known interatomic potentials, have made it possible to predict solubilities and related functions for atomic systems to an accuracy that is within experimental error, without resorting to any adjustable parameters. Also, by examining the structure of the terms in the perturbation expansions we gain insight into the atomic basis for the microscopic phenomena of cavitation and solvation. We can also determine the influence of non-classical statistical behavior and non-additivity of the pair potentials on solubility. Clearly, solubility theory for atomic systems has evolved to an accurate and sophisticated level.

Solubility theories for molecular systems are of course more primitive. This is because full, angle-dependent, intermolecular potentials are not known with any real accuracy for molecular systems and because molecular perturbation theories (or integral equation-based 

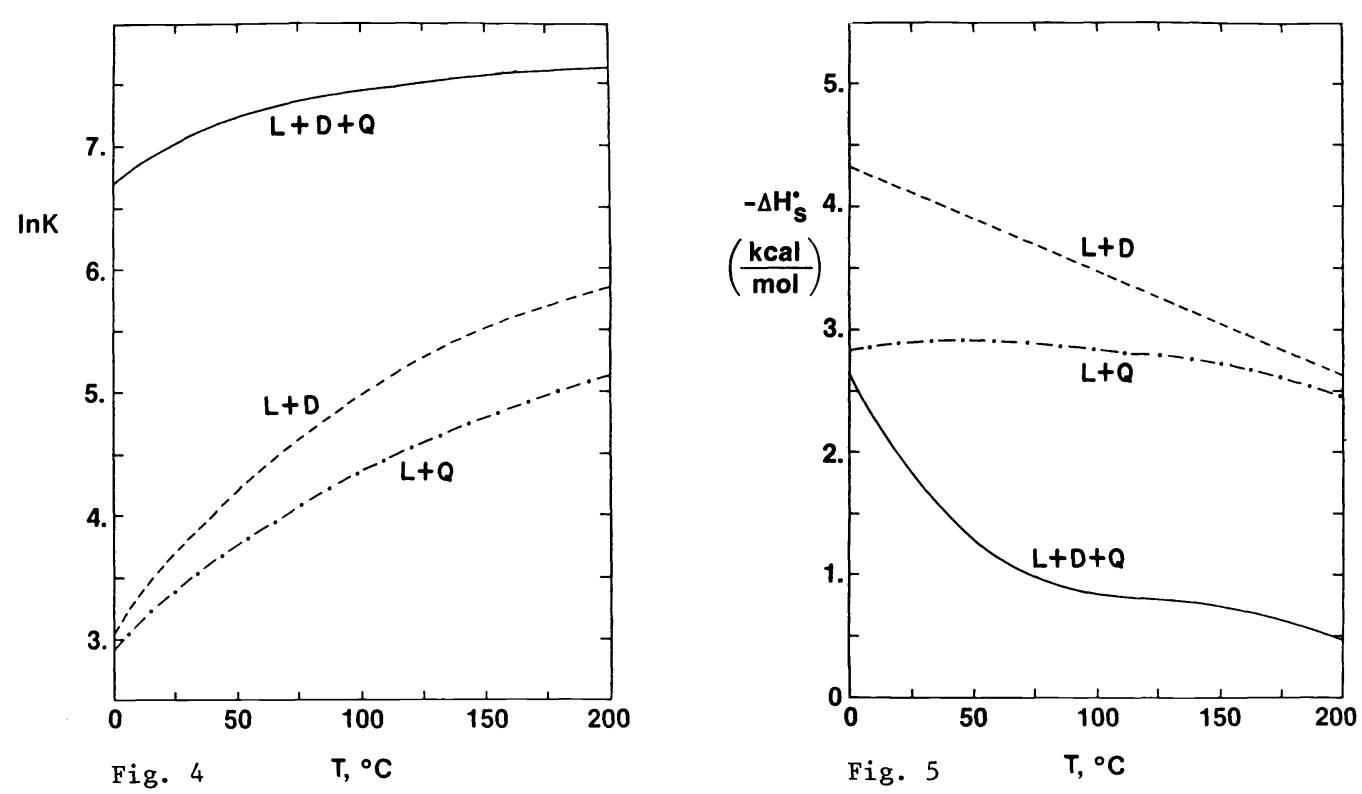

Fig. 4. The Henry's law constant $\mathrm{K}$ of a Lennard-Jones solute in the three hypothetical solvents versus temperature. $\mathrm{K}$ is based on pressure units in atmospheres. The solid, dashed, and alternating dot-dashed curves are for the fully generalized Stockmayer potential, the Stockmayer potential, and the LennardJones plus generalized quadrupole potential, respectively. For full details on the potentials, see Ref. 28. The solvent densities correspond to a total pressure of $1.0 \mathrm{~atm}$.

Fig. 5. The molar heat of solution at infinite dilution versus temperature. Solute, solvents, pressure, and legend are the same as in the caption to Fig. 4.
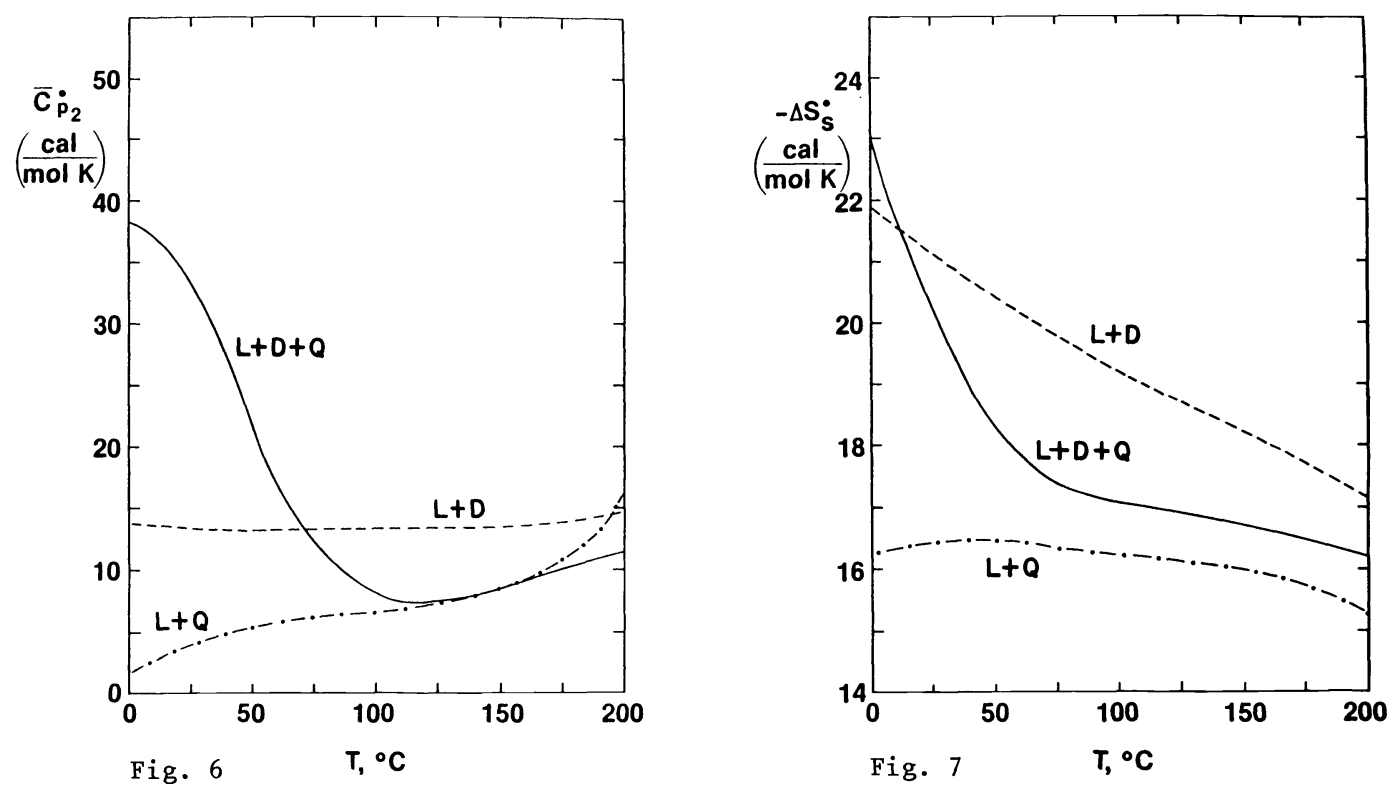

Fig. 6. The partial molal constant pressure heat capacity at infinite dilution versus temperature. Solute, solvents, pressure, and legend are the same as in the caption to Fig. 4.

Fig. 7. The molar entropy of solution at infinite dilution versus temperature. Solute, solvents, pressure, and legend are the same as in the caption to Fig. 4. 
theories) cannot yet accurately handle strong anisotropies in the pair potentials. Work on the latter problem is progressing well in a number of laboratories throughout the world. However, the extraction of the full angle-dependent pair potentials for the kinds of complex molecules that interest chemists remains a very difficult and long-term enterprise. In the meanwhile, much can still be learned in molecular systems by setting up models with specific anisotropies that can be handled theoretically, to act as probes to determine the salient molecular factors that influence solubility. An example of how this can be done was outlined in this article for electrostatic influences on non-electrolyte solubilities. Obviously, the influence of a variety of other types of anisotropies can be studied in a similar way.

Acknowledgement - This work was supported by a grant from the Natural Sciences and Engineering Research Council of Canada.

\section{REFERENCES}

1. B. Sisskind and I. Kasarnowsky, Z. Anorg. Allgem. Chem., 214, 385-395 (1933).

2. H.H. Uhlig, J. Phys. Chem., 41, 1215-1225 (1937).

3. D.D. Eley, Trans. Faraday Soc., 35, 1281-1293 (1939).

4. R.A. Pierotti, J.Phys. Chem., $6 \overline{7}, 1840-1845$ (1963).

5. H. Reiss, H.L. Frisch and J.L. Lebowitz, J. Chem. Phys., 31, 369-380 (1959). H. Reiss, H.L. Frisch, E. Helfand, and J.L. Lebowitz, ibid., 32, 119-124 (1960): E. Helfand, H. Reiss, H.L. Frisch, and J.L. Lebowitz, ibid., 33, $1379-1385$ (1960); H. Reiss, Adv. Chem. Phys., 9, 1-84 (1965).

6. L. Verlet, Phys. Rev., 165, 201-214 (1968).

7. R. Zwanzig, J. Chem. Phys., 22, 1420-1426 (1954).

8. J.A. Barker and D. Henderson, J. Chem. Phys., 47, 4714-4721 (1967).

9. P.J. Leonard, D. Henderson, and J.A. Barker, Trans. Faraday Soc., 66, 2439-2452 (1970).

10. G.A. Mansoori and F.B. Canfield, J. Chem. Phys., 51, 4958-4967 (1969).

11. G.A. Mansoori and T.W. Leland, Jr., J. Chem. Phys., 53, 1931-1936 (1970).

12. J.D. Weeks, D. Chandler, and H.C. Andersen, J. Chem. Phys., 54, 5237-5247 (1971).

13. L.L. Lee and D. Levesque, Mo1. Phys., 26, 1351-1370 (1973).

14. D. Henderson, J. Chem. Phys., 61, 926-931 (1974).

15. S.H. Sung, D. Chandler, and B.J. Alder, J. Chem. Phys., 61, 932-935 (1974).

16. T.L. Hill, An Introduction to Statistical Mechanics, p. 484, Addison-Wesley, Reading (1960)

17. D. Chandler, Acc. Chem. Res., 7, 247-251 (1974).

18. S. Goldman, J. Solution Chem., 6, 461-474 (1977).

19. D.A. McQuarrie, "Statistical Mechanics", Harper and Row, New York, 1976, pp 324.

20. S. Goldman, J. Phys. Chem., 81, 608-614 (1977).

21. R.0. Neff and D.A. McQuarrie, J. Phys. Chem., 77, 413-418 (1973).

22. S. Goldman, J. Chem. Phys., 67, 727-732 (1977).

23. D. Henderson, Annu. Rev. Phys. Chem., 25, 461-483 (1974).

24. S. Goldman, J. Chem. Phys., 69, 3775-3781 (1978).

25. B.M. Axilrod and E. Teller, J. Chem. Phys., 11, 299-300 (1943).

26. J.K. Lee, D. Henderson, and J.A. Barker, Mol. Phys., 29, 429-435 (1975).

27. P. Barnes, J.L. Finney, J.D. Nicholas, and J.E. Quinn, Nature (London) 282, 459-464 (1979).

28. S. Goldman, J. Chem. Phys., 75, 4064-4076 (1981).

29. J.A. Pople, Proc. R. Soc. London Ser. A 221, 508-516 (1954).

30. M.S. Ananth, K.E. Gubbins, and C.G. Gray, Mol. Phys., 28, 1005-1030 (1974).

31. G. Stel1, J.C. Rasaiah, and H. Narang, Mol. Phys., 27, 1393-1414 (1974).

32. M. Glytzani-Stephanopoulos, K.E. Gubbins, and C.G. Gray, Mo1. Phys., 30, 1649-1676 (1975).

33. C.H. Twu, K.E. Gubbins, and C.G. Gray, J. Chem. Phys., 64, 5186-5197 (1976).

34. C.G. Gray, K.E. Gubbins, and C.H. Twu, J. Chem. Phys.., 69, 182-193 (1978).

35. J.C.G. Calado, C.G. Gray, K.E. Gubbins, A.M.F. Palavra, V.A.M. Soares, L.A.K. Staveley, and C.H. Twu, J. Chem. Soc. Faraday Trans. 1, 74, 893-911 (1978).

36. P. Clancy, K.E. Gubbins, and C.G. Gray, Faraday Discuss. Chem. Soc., 66, 116-129 (1978).

37. S. Murad, K.E. Gubbins, and C.G. Gray, Chem. Phys. Lett., 65, 187-191 (1979).

38. J.J. Nicolas, K.E. Gubbins, W.B. Streett, and D.J. Tildesley, Mol. Phys., 37, 14291454 (1979).

39. E. Wilhelm, R. Battino, and R.J. Wilcock, Chem. Rev., 77, 219-262 (1977).

40. W.B. Streett, J. Chem. Phys., 42, 500-503 (1965).

41. W.B. Streett, J. Chem. Phys., $\frac{46}{46}, 3282-3286$ (1967).

42. E.B. Smith and J. Walkley, J. Phys. Chem., 66, 597-599 (1962).

43. J.H. Hildebrand and R.L. Scott, Regular Solutions, Prentice-Hall, New Jersey (1962).

44. M. Orentlicher and J.M. Prausnitz, Chem. Eng. Sci., 19, 775-782 (1964).

45. W.B. Streett, Cryogenics, 5, 27-33 (1965).

46. K.A. Solen, P.L. Chueh, and J.M. Prausnitz, Ind. Eng. Chem. Process Des. Dev., 9, 310317 (1970). 